\title{
Genetics and molecular biology: phospholipid transfer protein in atherogenesis
}

\author{
David Akopian and Jheem D. Medh \\ Department of Chemistry and Biochemistry, California State University at Northridge, Northridge, \\ California, USA
}

\begin{abstract}
Human phospholipid transfer protein (PLTP) is a 476 amino acid secreted protein that catalyzes the transfer of phospholipids and vitamin $E$ between lipoprotein particles $[1,2 \bullet, 3 \bullet]$. PLTP plays a key role in the formation and homeostasis of high-density lipoprotein (HDL) particles; it transfers phospholipids from very-low-density lipoprotein (VLDL) surface remnants to HDL particles, it facilitates the interconversion of HDL subclasses, and generates lipid-poor pre $\beta$ HDL particles. PLTP is synthesized by various cell types but its primary function is in the plasma where it is transported in association with apolipoproteins. Plasma PLTP exists in two forms: a high activity form that co-purifies with apolipoprotein (apo) $\mathrm{E}$ and a low activity form associated with apoA-I. It was reported recently that both apoE and apoA-IV can activate PLTP $[4 \bullet]$.
\end{abstract}

PLTP has both pro- and antiatherogenic characteristics. Pre $\beta$-HDL particles, a major product of PLTP catalysis, are required as acceptors for the atheroprotective cholesterol efflux pathway [5]. Studies in human subjects and genetically engineered mice indicate that plasma PLTP activity is proatherogenic. Human subjects with cardiovascular disease (CVD) have higher plasma PLTP activity compared with normal subjects [6]. Similarly, obesity and type 2 diabetes are coincident with high PLTP activity [7,8]. Overexpression of PLTP in $\mathrm{LDLR}^{-l-}$ mice results in a reduction in plasma HDL and apoA-I levels, an increase in VLDL secretion, and an increase in susceptibility to atherosclerosis despite lower apoB-containing lipoproteins [9]. Plasma HDL levels are also decreased in PLTP knock-out mice [10]. These mice, however, show a dramatic reduction in apoB synthesis and a significantly reduced susceptibility to diet-induced atherosclerosis.

\begin{abstract}
Several recent studies were aimed at better understanding the pro- and antiatherogenic mechanisms of PLTP activity. The current consensus is that systemic or plasma PLTP is proatherogenic, but macrophage PLTP is atheroprotective. Support for a proatherogenic role of plasma PLTP was obtained from a recent study investigating the relationship between PLTP activity and various inflammatory markers in humans with CVD [11•]. PLTP activity was positively and independently correlated with the main inflammatory marker C-reactive protein (CRP). Positive association of PLTP was also seen with other inflammation markers including serum amyloid A protein (SAA) and interleukin-6. Cardiovascular risk factors including body mass index, insulin resistance, apoE (VLDL), and apoB levels were directly correlated with PLTP activity as well as with CRP and SAA levels. These results clearly indicate that PLTP activity is important in both inflammation and CVD. Another marker of CVD, coronary artery intima-media thickness (IMT), was found to be independently associated with PLTP activity in diabetics, but not in nondiabetic subjects [12•]. ApoE may play a key role in the
\end{abstract}


pathophysiological effects of PLTP. In diabetic subjects, the level of active plasma PLTP appeared to be modulated by apoE [13•]. PLTP activity showed a strong positive correlation with plasma apoB and apoE levels, but was inversely related to apoA-I levels [13•]. As the active form of PLTP associates with apoE and the inactive form with apoA-I, these results suggest that apoE may be capable of converting inactive PLTP into the active form. The relationship between apoE and PLTP was further investigated in diabetic subjects of the Diabetes Atorvastatin Lipid Intervention (DALI) study [14•]. Atorvastatin treatment significantly reduced plasma apoE levels with a concomitant decrease in plasma PLTP activity. In a multivariate model, the change in apoE levels was the only determinant of the reduction in PLTP activity. Thus, apoE levels appear to modulate plasma PLTP activity.

The main function of PLTP is to modify and modulate HDL levels. Thus, plasma apoA-I and HDL levels are reduced in both PLTP over-expressing transgenic mice and in PLTP knockout mice $[9,10]$. As the liver is a primary source of plasma apoA-I, the hepatic synthesis and secretion of apoA-I was compared in wild-type and PLTP-deficient mice (PLTP-KO) [15••]. The level of apoA-I synthesis was not compromised in hepatocytes from PLTP knock-out mice. The lipidation of apoA-I was, however, defective in PLTP-KO hepatocytes such that the secreted particle contained fewer phospholipids and an altered composition of phosphatidylcholine, sphingomyelin, and fatty acyl groups. This phospholipid-deficient apoAI particle was less stable than the normally lipidated apoA-I particle derived from wild-type hepatocytes. Thus, the reduced HDL level in PLTP-deficient mice is due to increased degradation of apoA-I. Previous studies have already shown that stimulation of a proteolytic pathway is responsible for degradation of hepatic apoB in PLTP-deficient mice [16•].

The lower susceptibility to atherosclerosis seen in PLTP-deficient mice despite lower HDL levels is explained by the effect of peripheral PLTP at the cellular level. PLTP is responsible for the formation of pre $\beta$-HDL particles that serve as acceptors for ABCA1-mediated cholesterol efflux from macrophages [5]. The expression of both PLTP and ABCA1 in macrophages is induced by cholesterol-loading, a process mediated by the liver-X receptor [17]. Such coregulation suggests possible cooperative functions for PLTP and ABCA1. PLTP has previously been shown to bind to and stabilize cell surface ABCA1 [18]. Recently, a comparison of the cholesterol efflux capacities of peritoneal macrophages from wild-type and PLTP knock-out mice showed that lipid-free apoA-I was inefficient at removing cholesterol from cholesterol-loaded PLTP knock-out macrophages [19••]. Drastic upregulation of ABCA1, however, by treatment with cAMP abolished the difference between the efflux capacities of wild-type and PLTP knock-out macrophages. The nature of the cholesterol acceptor also contributed to the extent of efflux, as plasma from wild-type mice had a greater efflux potential than plasma from PLTP knock-out mice.

To examine the atheroprotective function of macrophage PLTP further, Valenta et al. [20] used bone marrow transplantation techniques to generate macrophage-specific deficiency of PLTP. In the $\mathrm{LDLR}^{-/-}$background, macrophage PLTP deficiency caused a significant increase in aortic lesion area without affecting plasma cholesterol levels. PLTP-deficient macrophages also showed a reduced antioxidative potential and increased oxidative stress as indicated by a dramatic decrease in cellular vitamin E content and a corresponding increase in intracellular hydroperoxides [20]. Thus, various mechanisms may exert the atheroprotective function of macrophage PLTP: cholesterol efflux may be enhanced due to the formation of pre $\beta$-HDL particles as cholesterol acceptors, PLTP may stabilize ABCA1, or the accumulation of vitamin E may lead to reduced oxidative capacity of macrophages. 


\section{Abbreviations}

apo, apolipoprotein; CRP, C-reactive protein; CVD, cardiovascular disease; HDL, highdensity lipoprotein; IMT, intima-media thickness; LDL, low-density lipoprotein; LDLR, lowdensity lipoprotein receptor; PLTP, phospholipid transfer protein; VLDL, very-low-density lipoprotein.

\section{References}

1. Jiang XC, Zhou HW. Plasma lipid transfer proteins. Curr Opin Lipidol 2006;17:302-308. [PubMed: 16680037]

2. Stein O, Stein Y. Lipid transfer proteins (LTP) and atherosclerosis. Atherosclerosis 2005;178:217230. [PubMed: 15694928]

3. Phillips SE, Vincent P, Rizzieri KE, et al. The diverse biological functions of phosphatidylinositol transfer proteins in eukaryotes. Crit Rev Biochem Mol Biol 2006;41:21-49. [PubMed: 16455519]

4. Janis MT, Metso J, Lankinen H, et al. Apolipoprotein E activates the low-activity form of human phospholipid transfer protein. Biochem Biophys Res Commun 2005;331:333-340. [PubMed: 15845396]

5. Von Exkardstein A, Jauhuaunen M, Huang Y, et al. Phospholipid transfer protein mediated conversion of high density lipoproteins generates prebeta 1-HDL. Biochim Biophys Acta 1996;1301:255-262. [PubMed: 8664337]

6. Schlitt A, Bickel C, Thumma P, et al. High plasma phospholipid transfer protein levels as a risk factor for coronary artery disease. Arterioscler Thromb Vasc Biol 2003;23:1857-1862. [PubMed: 12947020]

7. Riemens SC, van Tol A, Sluiter WJ, Dullaart RPF. Plasma phospholipid transfer protein activity is related to insulin resistance: impaired acute lowering by insulin in obese type II diabetic patients. Diabetologia 1998;41:929-934. [PubMed: 9726595]

8. Kaser S, Sandhofer A, Foger B, et al. Influence of obesity and insulin sensitivity on phospholipid transfer protein activity. Diabetologia 2001;44:1111-1117. [PubMed: 11596664]

9. Van Haperen R, van Tol A, van Gent T, et al. Increased risk of atherosclerosis by elevated plasma levels of phospholipid transfer protein. J Biol Chem 2002;277:48938-48943. [PubMed: 12372822]

10. Jiang XC, Qin S, Qiao C, et al. Apolipoprotein B secretion and atherosclerosis are decreased in mice with phospholipid transfer protein deficiency. Nat Med 2001;7:847-852. [PubMed: 11433351]

11. Cheung MC, Brown BG, Marino Larsen EK, et al. Phospholipid transfer protein activity is associated with inflammatory markers in patients with cardiovascular disease. Biochim Biophys Acta 2005;1762:131-137. [PubMed: 16216472]

12. De Vries R, Dallinga-Thie GM, Smit AJ, et al. Elevated plasma phospholipid transfer protein activity is a determinant of carotid intima-media thickness in type 2 diabetes mellitus. Diabetologia 2006;49:398-404. [PubMed: 16374627]

13. Tan KC, Shiu SW, Wong Y, et al. Plasma apolipoprotein E concentration is an important determinant of phospholipid transfer protein activity in type 2 diabetes mellitus. Diabetes Metab Res Rev 2006;22:307-312. [PubMed: 16389649]

14. Dallinga-Thie GM, van Tol A, Hattori H, et al. Plasma phospholipid transfer protein activity is decreased in Type 2 diabetes during treatment with atorvastatin: A role for apolipoprotein E? Diabetes 2006;55:1491-1496. [PubMed: 16644710]

15. Siggins S, Bykov I, Hermansson M, et al. Altered hepatic lipid status and apolipoprotein A-I metabolism in mice lacking phospholipid transfer protein. Atherosclerosis. 2006[Epub ahead of print]

16. Jiang XC, Li Z, Liu R, et al. Phospholipid transfer protein deficiency impairs apolipoprotein-B secretion from hepatocytes by stimulating a proteolytic pathway through a relative deficiency of vitamin E and an increase in intracellular oxidants. J Biol Chem 2005;280:18336-18340. [PubMed: 15734742]

17. Laffitte BA, Joseph SB, Chen M, et al. The phospholipid transfer protein gene is a liver X receptor target expressed by macrophages in atherosclerotic lesions. Mol Cel Biol 2001;23:2182-2191. 
18. Oram JF, Wolfbauer G, Vaughan AM, et al. Phospholipid transfer protein interacts with and stabilizes ATP-binding cassette transporter A1 and enhances cholesterol efflux from cells. J Biol Chem 2003;278:52379-52385. [PubMed: 14559902]

19. Lee-Rueckert M, Vikstedt R, Metso J, et al. Absence of endogenous phospholipid transfer protein (PLTP) impairs ABCA1-dependent efflux of cholesterol from macrophage foam cells. J Lipid Res. 2006[Epub ahead of print]

20. Valenta DT, Ogier N, Bradshaw G, et al. Atheroprotective potential of macrophage-derived phospholipid transfer protein in low-density lipoprotein receptor-deficient mice is overcome by apolipoprotein AI overexpression. Arterioscler Thromb Vasc Biol 2006;26:1572-1578. [PubMed: 16675720]

\section{Recommended reading}

21. Aouizerat BE, Engler MB, Natanzon Y, et al. Genetic variation of PLTP modulates lipoprotein profiles in hypoalphalipoproteinemia. J Lipid Res 2006;47:787-793. [PubMed: 16388083] A relatively large sample (364 controls and 276 subjects with hypoalphalipoproteinemia) was used for the first time to conduct a sequence analysis of the PLTP gene with the purpose of identifying sequence anomalies and their associations with hypoalphalipoproteinemia. The authors discovered 18 variations of the PLTP gene, one of which was the c. $-34 \mathrm{G}>\mathrm{C}$ polymorphism. The presence of the $\mathrm{c} .-4 \mathrm{G}>\mathrm{C}$ minor allele was found to have an antiatherogenic effect on plasma tryglicerides, HDL-C, and trygliceride/ HDL-C profiles, pointing to the role of PLTP in cardiovascular disease. Further studies involving sequence variations in the PLTP gene and their effect on plasma lipids are suggested in this report.

22. Bosse Y, Bouchard L, Despres J-P, et al. Haplotypes in the phospholipid transfer protein gene are associated with obesity-related phenotypes: the Quebec Family Study. Int J Obes 2005;29:13381345. This is the first study to demonstrate the association between PLTP genetic variants and obesity. The authors observed an association between the c. $-87 \mathrm{G}>\mathrm{A}$ polymorphism in intron 1 and obesityrelated phenotypes, with AT haplotype being positively associated with obesity. Unlike the c. $-87 \mathrm{G}>\mathrm{A}$ polymorphism, the c. $1175+68 \mathrm{~T}>$ polymorphism was not associated with obesity-related phenotypes. Interestingly, sequencing the promoter and the coding region of the PLTP gene did not reveal any mutations that produce the same results.

23. Bunte H, Schenning M, Sodaar P, et al. A phosphatidylinositol transfer protein $\alpha$-dependent survival factor protects cultured primary neurons against serum deprivation-induced cell death. J Neurochem 2006;97:707-715. [PubMed: 16573656] The role of phosphatidylinositol transfer protein $\alpha$ (PI-TP $\alpha)$ in protecting primary neurons from serum deprivation-induced cell death was investigated in this study. The authors report a significant increase in survival of the neurons incubated in the conditioned medium from fibroblasts overexpressing PI-TP $\alpha$. Based on the findings in this study, a hypothetical pathway of the PI-TP $\alpha$-dependent production of the survival factor and its action through a $\mathrm{G}$ protein linked receptor is proposed.

24. Cheung MC, Albers JJ. Active plasma phospholipid transfer protein is associated with apoA-I- but not apoE-containing lipoproteins. J Lipid Res 2006;47:1315-1321. [PubMed: 16520487] Using antiA-I, antiA-II, and antiE immunoadsorbents to remove plasma PLTP and measure the activity of PLTP, the authors of this study found that, contrary to the previous findings, the active form of PLTP is associated with apoA-I rather than apoE. The explanation is provided as to how the use of dextran sulfate cellulose column for the removal of apoE and apoB could lead to the conclusion that the active form of PLTP is associated with apoE.

25. Cheung MC, Brown BG, Martino Larsen EK, et al. Phospholipid transfer protein activity is associated with inflammatory markers in patients with cardiovascular disease. Biochim Biophys Acta 2006;1762:131-137. [PubMed: 16216472] The authors of this report investigated the relationships of the risk factors of CVD with PLTP and five inflammatory markers, CRP, serum amyloid A protein, interleukin-6, white blood cells, and fibrinogen, in 93 patients with CVD. Among positive associations observed, of special interest is a strong positive correlation between plasma PLTP activity and CRP, a marker of inflammation. This study suggests that PLTP plays a role in atherosclerosis, a chronic state of inflammation, and metabolic syndrome. Anti- and proatherogenic effects of PLTP are briefly discussed in the paper.

26. Dallinga-Thie GM, van Tol A, Hattori H, et al. Plasma phospholipid transfer protein activity is decreased in type 2 diabetes during treatment with atorvastatin: a role for apolipoprotein E? Diabetes 
2006;55:1491-1496. [PubMed: 16644710] In this study, the effect of a statin on plasma PLTP activity was investigated for the first time in patients with type 2 diabetes. Atorvastatin decreased plasma PLTP activity and dose-dependently increased total plasma PLTP mass. The decrease in PLPT activity strongly correlated with the decrease in plasma levels of apolipoprotein E (apoE). The results of the study are in agreement with the reports suggesting that the active form of PLTP is associated with apoE. The authors suggest that atorvastatin inactivates plasma PLTP by destabilizing apoE.

27. de Vries R, Dallinga-Thie GM, Smit AJ, et al. Elevated plasma phospholipid transfer protein activity is a determinant of carotid intima-media thickness in type 2 diabetes mellitus. Diabetologia 2006;49:398-404. [PubMed: 16374627] The effect of plasma PLTP activity on carotid artery intimamedia thickness (IMT) was investigated in 87 subjects with type 2 diabetes mellitus and 83 controls. The authors report an independent positive association between plasma PLTP activity and IMT in subjects with type 2 diabetes mellitus. This study suggests that increased PLTP activity in patients with type 2 diabetes mellitus is proatherogenic and can be used as a marker of the cardiovascular disease.

28. Janis MT, Metso J, Lankinen H, et al. Apolipoprotein E activates the low-activity form of human phospholipid transfer protein. Biochem Biophys Res Commun 2005;331:333-340. [PubMed: 15845396] Using surface plasmon resonance analysis, the authors of this study showed that while both low- and high-activity forms of PLTP bind apoE, apoA-I, and apoA-IV, the binding affinities and rates are different for each interaction. Binding of apoE and apoA-IV but not apoA-I was found to concentration-dependently activate low-activity PLTP. This finding is contradictory to the results reported by Cheung et al., who showed that active PLTP is associated with apoA-I rather than apoE.

29. Jiang X, Li Z, Liu R, et al. Phospholipid transfer protein deficiency impairs apolipoprotein-B secretion from hepatocytes by stimulating a proteolytic pathway through a relative deficiency of vitamin $\mathrm{E}$ and an increase in intracellular oxidants. J Biol Chem 2005;280:18336-18340. [PubMed: 15734742] The mechanism of PLTP-dependent impairment of apolipoprotein-B (apoB) secretion from hepatocytes was investigated in this study, using PLTP knock-out apoB transgenic mice. PLTP deficiency caused a decrease in liver vitamin E content, an increase in liver peroxide content, and reactive oxygen species-dependent degradation of apoB through post-estrogen receptor presecretory proteolysis pathway. Impaired secretion of apoB, a major constituent of atherogenic lipids, in PLTP-deficient mice suggests that inhibition of PLTP activity may be antiatherogenic.

30. Lee-Rueckert M, Vikstedt R, Metso J, et al. Absence of endogenous phospholipid transfer protein (PLTP) impairs ABCA1-dependent efflux of cholesterol from macrophage foam cells. J Lipid Res. 2006[Epub ahead of print] It is well known that exogenous PLTP stimulates ABCA1-dependent cholesterol efflux from cultured macrophages. In this study, the role of the endogenous PLTP in ABCA1-dependent cholesterol efflux was investigated using peritoneal macrophages from wild-type and PLTP knock-out mice. The authors found that the absence of the endogenous PLTP impairs ABCA1-mediated cholesterol efflux from the cholesterol-loaded macrophages. The difference in the efflux of cholesterol from the PLTP-deficient and wild-type cells, however, can be erased by treating the cells with cAMP. This demonstrates that endogenous PLTP is required for efficient reverse cholesterol transport and therefore plays an atheroprotective role.

31. Lie J, Lankhuizen IM, Gross B, et al. Fenofibrate reverses the decline in HDL cholesterol in mice overexpressing human phospholipid transfer protein. Biochim Biophys Acta 2005; 1738:48-53. [PubMed: 16298159] The effect of fenofibrate on PLTP expression and activity and plasma HDL levels was investigated using cultured primary human hepatocytes, wild-type mice, and transgenic mice overexpressing human PLTP. Previous reports show that increased PLTP activity lowers plasma HDL levels. The authors of this study conclude that fenofibrate counteracts this effect of increased PLTP activity and suggest that its use may be beneficial in patients with disorders characterized by increased PLTP activity such as type 2 diabetes mellitus.

32. Oomen PHN, van Tol A, Hattori H, et al. Human plasma phospholipid transfer protein activity is decreased by acute hyperglycaemia: studies without and with hyperinsulinaemia in type 1 diabetes mellitus. Diabet Med 2005;22:768-774. [PubMed: 15910630] The independent effect of hyperglycemia and hyperinsulinemia on plasma PLTP mass and activity was studied for the first time in 16 patients with type 1 diabetes mellitus. While separately hyperglycemia and hyperinsulinemia did not significantly affect PLTP mass, the combined effect of these conditions was the reduction in PLTP mass. Both hyperglycemia and hyperinsulinemia, however, independently caused a decrease 
in plasma PLTP activity. These results are contradictory to the previous findings according to which plasma PLTP levels are upregulated by hyperglycemia.

33. Phillips SE, Vincent P, Rizzieri KE, et al. The diverse biological functions of phosphatidylinositol transfer proteins in eukaryotes. Crit Rev Biochem Mol Biol 2006;41:21-49. [PubMed: 16455519] This is an in-depth review detailing the structure and functions of phosphatidylinositol transfer proteins (PITPs) in yeast, plants, and mammals. A considerable portion of the review focuses on the structure and biological roles of Sec14p PITP of Saccharomyces cerevisiae. Here, the authors present the most recent findings and discuss the role of other proteins involved in signaling and lipid metabolic pathways requiring Sec14p. Mammalian and plant PITPs are also discussed in detail. The importance of further research in the area of PITPs is highlighted through the discussion of the questions that demand investigation.

34. Tan KCB, Shiu SWM, Wong Y, Tam S. Plasma phospholipid transfer protein activity and subclinical inflammation in type 2 diabetes mellitus. Atherosclerosis 2005;178:365-370. [PubMed: 15754464] Independent positive correlation between CRP, a marker of inflammation, and lasma PLTP activity was observed in patients with type 2 diabetes mellitus recruited for the study of the relationship between subclinical inflammation and plasma PLTP activity. Although the authors show that subclinical inflammation may influence PLTP activity, the report does not establish causal relationship between PLTP activity and CRP.

35. Tan KCB, Shiu SWM, Wong Y, et al. Plasma apolipoprotein E concentration is an important determinant of phospholipid transfer protein activity in type 2 diabetes mellitus. Diabetes Metab Res Rev 2006;22:307-312. [PubMed: 16389649] The authors of this report studied the association of plasma apolipoprotein levels with plasma PLTP activity in patients with type 2 diabetes mellitus and control subjects. A significant positive correlation was found between apoE and apoA-I concentration and plasma PLTP activity in diabetic patients and controls. Plasma PLPT activity was found to be determined mainly by the levels of apoE in patients with type 2 diabetes mellitus. The study suggests that apolipoproteins E and A-I are important in influencing plasma PLTP activity.

36. Schlitt A, Liu J, Yan D, et al. Anti-inflammatory effects of phospholipid transfer protein (PLTP) deficiency in mice. Biochim Biophys Acta 2005;1733:187-191. [PubMed: 15863365] This definitive study shows that PLTP deficiency is associated with anti-inflammatory state in PLTP knock-out mice. Based on the comparison of the levels of interleukin-6 (IL-6), a known regulator of the production of inflammatory cytokines, and the ability of exogenous IL-6 to induce inflammatory state in PLTP knock-out and wild-type mice, the authors conclude that PLTP plays an important role in inflammation and possesses proatherogenic properties.

37. Siggins S, Bykov I, Hermansson M, et al. Altered hepatic lipid status and apolipoprotein A-I metabolism in mice lacking phospholipid transfer protein. Atherosclerosis. 2006[Epub ahead of print] In this study the authors conducted in-vivo experiments using PLTP-deficient and wild-type mice as well as primary hepatocytes from these animals to investigate the role of PLTP in influencing the levels of plasma lipoproteins and the effect of PLTP deficiency on hepatic lipid status and apoA-I biosynthesis. PLTP-deficient animals exhibited lower HDL levels, altered VLDL and hepatic lipid composition, and were characterized by decreased stability of apoA-I. The report suggests that the role of PLTP in lipid metabolism is multifaceted.

38. Siggins S, Ehnholm C, Jauhiainen M, Olkkonen VM. Plasma phospholipid transfer protein fused with green fluorescent protein is secreted by HepG2 cells and displays phosphatidylcholine transfer activity. Biochem Cell Biol 2006;84:117-125. [PubMed: 16609691] The authors of this study fused the carboxy terminus of PLTP with green fluorescent protein using a 17 amino acids long spacer. The translocation of the fused protein within the biosynthetic pathway and its activity were studied. The fused protein exhibited a decreased specific activity compared with the endogenously expressed protein, demonstrating the importance of the C-terminal tail in phospholipid transfer activity. The use of the fused protein in studying the functions of PLTP and possible modifications of the construct for future experiments are discussed.

39. Stein O, Stein Y. Lipid transfer proteins (LTP) and atherosclerosis. Atherosclerosis 2005;178:217230. [PubMed: 15694928] This is a comprehensive review summarizing the current knowledge concerning four lipid transfer proteins: acyl-CoA:cholesterol acyltransferase, lecithin cholesterol acyltransferase, cholesteryl ester transfer protein, and phospholipid transfer protein. The results of the experiments with gene knock-out animal models, enzyme inhibitors, as well as the epidemiological and clinical studies are presented. 
40. Tremblay JM, Unruh JR, Johnson CK, Yarbrough LR. Mechanism of interaction of PITP $\alpha$ with membranes: Conformational changes in the C-terminus associated with membrane binding. Arch Biochem Biophys 2005;444:112-120. [PubMed: 16309627] Conformational changes in phosphatidylinositol transfer protein $\alpha$ (PITP $\alpha$ ) associated with the binding of the protein to phospholipid vesicles were investigated in this study by using PITP $\alpha$ derivitized with a fluorescent probe at several Cys residues of the $\mathrm{C}$-terminal helix and distal to it. The data suggest a significant conformational change in the C-terminal helix possibly involving its complete melting upon binding to the phospholipids vesicles. This finding is in agreement with the previous reports suggesting the importance of the $\mathrm{C}$-terminus of PITP $\alpha$ in binding to membranes.

41. Valenta DT, Ogier N, Bradshaw G, et al. Atheroprotective potential of macrophage-derived phospholipid transfer protein in low-density lipoprotein receptor-deficient mice is overcome by apolipoprotein AI overexpression. Atheroscler Thromb Vasc Biol 2006;26:1572-1578. The authors of this study introduced PLTP-deficient or PLTP-expressing bone marrow cells into LDL receptordeficient mice that either expressed mouse apoA-I or overexpressed human apoA-I with the purpose of investigating the role of the macrophage-derived PLTP in atherosclerosis. The presence of PLTPexpressing macrophages in mice with normal levels of apoA-I resulted in smaller atherosclerotic lesions compared with the mice that lacked the macrophage-derived PLTP. PLTP-deficient bone marrow cells had a lower vitamin E content, higher concentration of cellular hydroperoxides, and a higher capacity to oxidize exogenous LDL than PLTP-expressing cells. The study shows that, unlike plasma PLTP, the macrophage-derived PLTP is atheroprotective.

42. Vance JE, Vance DE. Metabolic insights into phospholipid function using gene-targeted mice. J Biol Chem 2005;280:10877-10880. [PubMed: 15642727] The latest findings concerning the genes involved in intracellular transport and metabolism of biologically active phospholipids, phosphatidylethanolamine, phosphatidylcholine, and phosphatidylserine, are summarized in this short review. The authors address the functions of some of the enzymes and transporters involved in phospholipid metabolism by discussing the results of the experiments with knock-out mice and ask important questions that suggest the direction of future research.

43. Wu MK, Cohen DE. Phosphatidylcholine transfer protein regulates size and hepatic uptake of highdensity lipoproteins. Am J Physiol Gastrointest Liver Physiol 2005;289:1067-1074. The role of phosphatidylcholine transfer protein (PC-TP) in HDL metabolism was investigated in this study. PCTP deficiency in PC-TP knock-out mice resulted in higher plasma lipid content, accumulation of smaller and denser HDL particles, and reduced uptake of HDL by the liver. Interestingly, PC-TP deficiency did not affect ABCA1 mRNA or protein levels; nor did it influence plasma total phospholipid content. 